\title{
Biological Control of
}

\section{Honey Bee Pests}

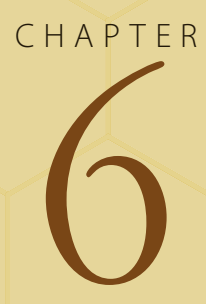

W. G. Meikle, D. Sammataro, and G. Mercadier

\section{Introduction}

Biological control is considered one of the pillars of Integrated Pest Management (IPM), yet it is seldom considered with respect to the management of bee pests. Part of the reason for that likely stems from biological control lying outside "traditional" bee pest management strategies, and partly from feeling that the logic of controlling an undesirable arthropod (mite or insect) within the environment of a highly desirable arthropod (the honey bee) is suspect. In addition, honey bees are notoriously fastidious and live in a highly sophisticated environment (the hive). Many researchers have no doubt felt that natural enemies, be they parasitoids, predators, or pathogens, would have a very difficult time establishing themselves.

Biological control is defined as pest control using natural enemies, whether those natural enemies are arthropods or microbes, and includes predators, parasites and parasitoids, and pathogens (Perkins and Garcia, 1999). Biological control has been used in many agricultural systems with great success (Gutierrez et al., 1999). There are two major kinds of biological control:

1. Classical biological control, in which a new organism such as a parasite or a pathogen is released into an ecosystem where it did not previously occur in order to control a particular pest.

2. Augmentative biological control, in which the natural enemy to be used already exists in the ecosystem but at a density too low to sufficiently control the target pest (Perkins and Garcia, 1999).

Augmentative biological control includes inoculative control, in which small numbers or quantities of natural enemies are released that subsequently increase their density naturally, and inundative control, in which a very high density of natural enemies is applied to ensure a high kill rate.
Abstract Biological control of bee pests is a small but growing field as beekeepers and bee researchers seek ways to reduce pesticide use. Of the arthropod pests of honey bees, those that have been targets of biological control on at least the laboratory level are the Wax Moths Galleria mellonella and Achroia grisella, the Varroa mite Varroa destructor, and the Small Hive Beetle Aethina tumida. Several organisms have been proposed as biological control agents against wax moth, including naturallyoccurring parasitoids, and one, Bacillus thuringiensis, has been commercialized. Biological control of $V$. destructor has involved application of entomopathogenic fungi, and while some results have been encouraging, more work is clearly needed with respect to isolate selection, formulation, and application method. Fungal agents have likewise been used against A. tumida and elevated mortality has been observed, but no field tests have been reported thus far. The interaction of biological control agents, bees, and target pests needs further research. 


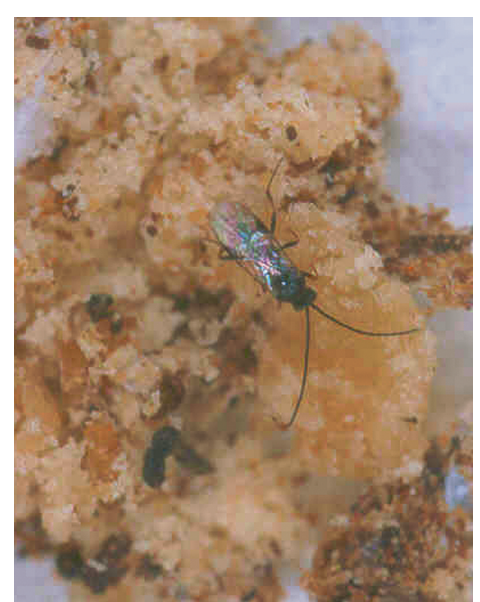

Figure 1. Apanteles galleriae Wilkinson, a wax moth parasitoid, lays its eggs in the larvae of wax moth. (Photo by D. Sammataro.)
The most likely targets for biological control are other arthropods, particularly the Greater Wax Moth (Galleria mellonella L.) and Lesser Wax Moth (Achroia grisella F.) (both Lepidoptera: Pyralidae); the Varroa mite (Varroa destructor Anderson and Trueman) (Mesostigmata: Varroidae); and the Small Hive Beetle (SHB) (Aethina tumida Murray) (Coleoptera: Nitidulidae). While the bee pests listed above are among the most important worldwide, they are not the only ones. Pests such as predatory wasps can cause considerable damage to bee hives; Vespa velutina (Hymenoptera: Vespidae) is an invasive species in France (Perrard et al., 2009). However, because these wasps do not live inside the hive environment, their control is implemented differently and has different constraints, and they will not be considered here. Mites such as Tropilaeleps spp. (Mesostigmata: Laelapidae) are not (yet) widespread enough in areas where the Western honey bee, Apis mellifera, occurs to have been the subject of many alternative control strategies. Similarly, biological control of Tracheal mites (Acarapis woodii [Rennie]) (Prostigmata: Tarsonemidae) is largely unexplored.

\section{Wax Moth}

Both G. mellonella and A. grisella have a number of natural enemies, including parasitoids and pathogens. Bollhalder (1999) proposed the use of an egg parasitoid, Trichogramma sp. (Hymenoptera: Trichogrammatidae). Larval parasitoids, however, have been examined more extensively. For example, Apanteles galleriae Wilkinson (Hymenoptera: Braconidae) is known to attack G. mellonella and several species of Achroia (Uçkan et al., 2004; Whitfield et al., 2001) (see Figure 1). Schöller and Prozell (2001) tested another braconid parasitoid, Habrobracon hebitor, against both $G$. mellonella and A. grisella as a potential commercial biocontrol agent to protect stored wax comb. Like A. galleriae, $H$. hebitor is a generalist parasitoid known to attack many species of moths. Blumberg and Ferkovich (1994) reported that Microplitis croceipes (Cresson), a braconid parasitoid whose usual host is the corn earworm Helicoverpa zea (Boddie) (Lepidoptera: Noctuidae), could reproduce successfully on G. mellonella as well. Harvey and Vet (1997) used G. mellonella as a host for Venturia canescens (Grav.) (Hymenoptera: Ichneumonidae).

If wax moths are suitable hosts to a number of parasitoid natural enemies in most if not all regions where they occur, then the question presents itself why the wax moth is not a problem. The answer may have to do with where the wax moth is found: inside silk and frass tunnels in honey bee comb (Figure 2). Honey bee worker activity likely keeps many parasitoids at bay and even those that get through must find and attack the larvae, which in healthy hives are at a low density. Uçkan et al. (2004) reported that $A$. galleriae was sensitive to high parasitoid densities and, in the absence of unattacked wax moth larvae, would hyperparasitize (i.e., attack parasitized) larvae, thus reducing the reproductive success of the parasitoids.

Bacterial products involving Bacillus thuringiensis (Bt) have been developed and tested against G. mellonella (Burges and Bailey, 1968), and come in two types: those involving the living bacteria (e.g., Burges and Bailey, 1968; Vandenberg and Shimanuki, 1990; Ellis and Hayes, 2009) and those involving just the endotoxin component of the bacteria (Burges and Bailey, 1968). Endotoxin products are not biological control in the usual sense because the active ingredient is not a living organism, but 
they are often described as "biopesticides." Tests using the live bacteria showed good protection of comb and low risk for bees (Burges and Bailey, 1968; Vandenberg and Shimanuki, 1990). Ellis and Hayes (2009) reported that comb made from foundation treated with $\mathrm{Bt}$ had 30\% higher mortality of wax moth larvae than untreated comb a week after treatment. They qualified this by pointing out that the cost and labor associated with applying $\mathrm{Bt}$ were likely not offset by the benefits. Commercial products made with the bacillus included Certan $^{\circledR}$, which is available once more from Internet sites under the B401 Certan ${ }^{\circledR}$ label. This product was sprayed onto stored combs to protect them from wax moth. In commercial beekeeping operations, where thousands of honey supers were stored, it can be an important control measure.

Wax moths, particularly G. mellonella, are susceptible to many pathogenic fungi and nematodes (Chandler et al., 1997), probably because many of these pathogens are soil-dwelling and wax moths are seldom exposed to the soil environment. Indeed, their susceptibility is such that Zimmerman (1986) developed the "Galleria bait method," which involves simply exposing the larvae to soil to search for entomopathogenic fungi (EPF). EPF are recovered then from the infected larvae. This technique has been used by others for both fungi and nematodes (e.g., Chandler et al., 1997). Because G. mellonella is acceptable as a host to such a wide range of pathogens, it is often used as a factitious host in studies on pathogen ecology. Spence et al. (2011) used G. mellonella as a host for three species of entomopathogenic nematodes, Heterorbabditis bacteriophora, Steinernema carpocapsae, and S. riobrave; and Koppenhöffer and Fuzy (2008) used it as a host for four nematode species: S. scarabaei, S. glaseri, H. zealandica, and H. bacteriophora. To date, nematodes have not been reported as biocontrol agents against wax moths.

Galleria mellonella has been used extensively in studies on EPF. Shapiro-Ilan et al. (2003) used G. mellonella larvae to explore for naturally occurring insect pathogens in pecan orchards and, in addition to several nematode species, they recovered Metarhizium anisopliae (Metschnikoff) Sorokin (Hypocreales: Clavicipitaceae) and Beauveria bassiana (Balsamo) Viullemin (Hypocreales: Cordycipitaceae) from larval cadavers. Tseng et al. (2008) used G. mellonella to examine infection by the fungus Nomuraea rileyi (Hypocreales: Clavicipitaceae). However, little work has been done to date on developing a biological control program against wax moths using either nematodes or fungi. A nuclear polyhedrosis virus, GmMNPV, was obtained from G. mellonella (Shapiro, 2000), and a number of alphanodaviruses replicate well in G. mellonella larvae (Johnson et al., 2000), but, as with nematodes and fungi, these viruses have not been explored to date as biological control agents.

Wax moths have many natural arthropod and microbial enemies but few biological control options are currently available. This is probably due largely to the expense of developing and producing the products, and to the availability of low-cost approaches for most situations, such as removing infested comb from hives and freezing stored comb (Charrière and Imdorf, 1999). Wax moths are problematic for weaker hives (Nielsen and Bister, 1979) but are generally not counted among the worst problems in apiculture except for commercial beekeepers who typically have to 


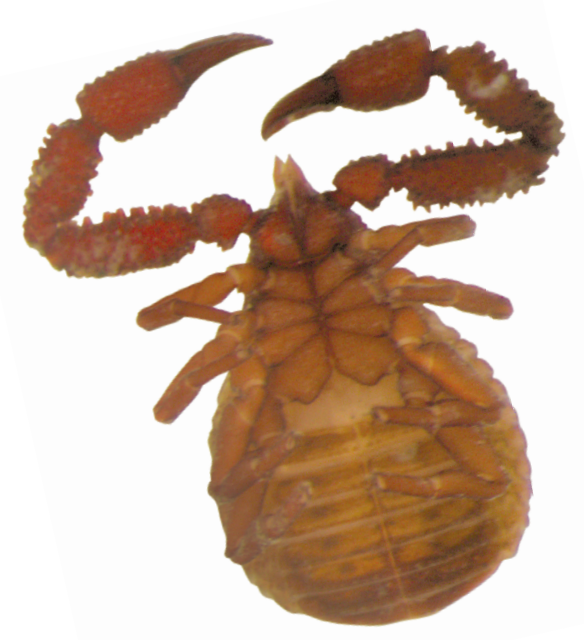

Figure 3. An unidentified pseudoscorpion, collected from an African bee colony in Kenya. Length of body is $6.35 \mathrm{~mm}$.

(Photo by D. Sammataro.) store thousands of empty hive bodies. Annual losses can be upwards of $\$ 5$ million (MAAREC, 2000). In most cases, chemical or thermal controls are used in their storage facilities.

\section{Varroa Mite}

Chandler et al. (2001) reviewed a wide range of taxa as candidate biological control agents against $V$. destructor. While parasitoids of $V$. destructor are unknown, the candidates did include a predator, pseudoscorpions (Pseudoscorpionida) (see Figure 3). Donovan and Paul (2005) suggested that the use of pseudoscorpions should be explored further because they are found naturally associated with feral honey bee colonies. Given that pseudoscorpions are active predators that would seek out mites, they probably are most affective against mites crawling on the comb. Pseudoscorpions would likely have little effect on phoretic mites or, owing to bee hygienic behavior, mites in brood cells, but they might impact mites on the comb. However, field evaluations have not been conducted as of this writing and the specificity is unknown.

Pathogens, especially bacteria and fungi, have been considered as potential biological control agents of $V$. destructor. Kleespies et al. (2000) reported a virus from a Varroa mite but no further work has been reported. Bacteria or bacterial products have also been evaluated and although the work did not always involve biological control in a strict sense, we include it here. Tu et al. (2010) isolated protein chitinases from a bacterium, Serratia marcescens, itself collected from the guts of the Asian honey bee workers (Apis cerana). They found that bees were not affected by the application of chitinases or the presence of chitinases in their food, and proposed the chitinases as a basis for genetically modified bee strains. Tsagou et al. (2004) isolated bacterial strains from $V$. destructor belonging to Bacillaceae (Bacillus sp.) and Micrococcaceae, in addition to three unidentified strains. The effect of these bacteria as whole cells, extracellular broth, and cellular extract, was tested on Varroa in laboratory bioassays. One Bacillus isolate in particular was found to decrease mite survivorship markedly. The bacteria have neither been tested for their effects on honey bees nor used in field trials.

\section{Fungi}

Most research on biological control of $V$. destructor has focused on the use of EPF, specifically facultative pathogens of the order Hypocreales. These fungi are preferred because they can be highly virulent against target pests, are easy to grow in vitro, and produce comparatively resistant spores called "conidia" that are easy to incorporate into a biopesticide (Goettel and Inglis, 1997). Davidson et al. (2003) examined growth rates of EPF in the genera Beauveria (see Figure 4A), Hirsutella, Metarhizium (see Figure 4B), Paecilomyces (now Isaria), Tolypocladium, and Verticillium in order to identify isolates which grew well at the higher temperatures encountered in bee hives. Shaw et al. (2002) reported the results of bioassays of isolates from these same genera-and many of the same isolates-against $V$. destructor and found several candidate isolates; no field tests were conducted. Published field trials have been largely confined to two species: M. anisopliae and B. bassiana. Both species have been used in other crop systems (Jaronski, 2010); neither are specific for $V$. destructor or even mites in general. 

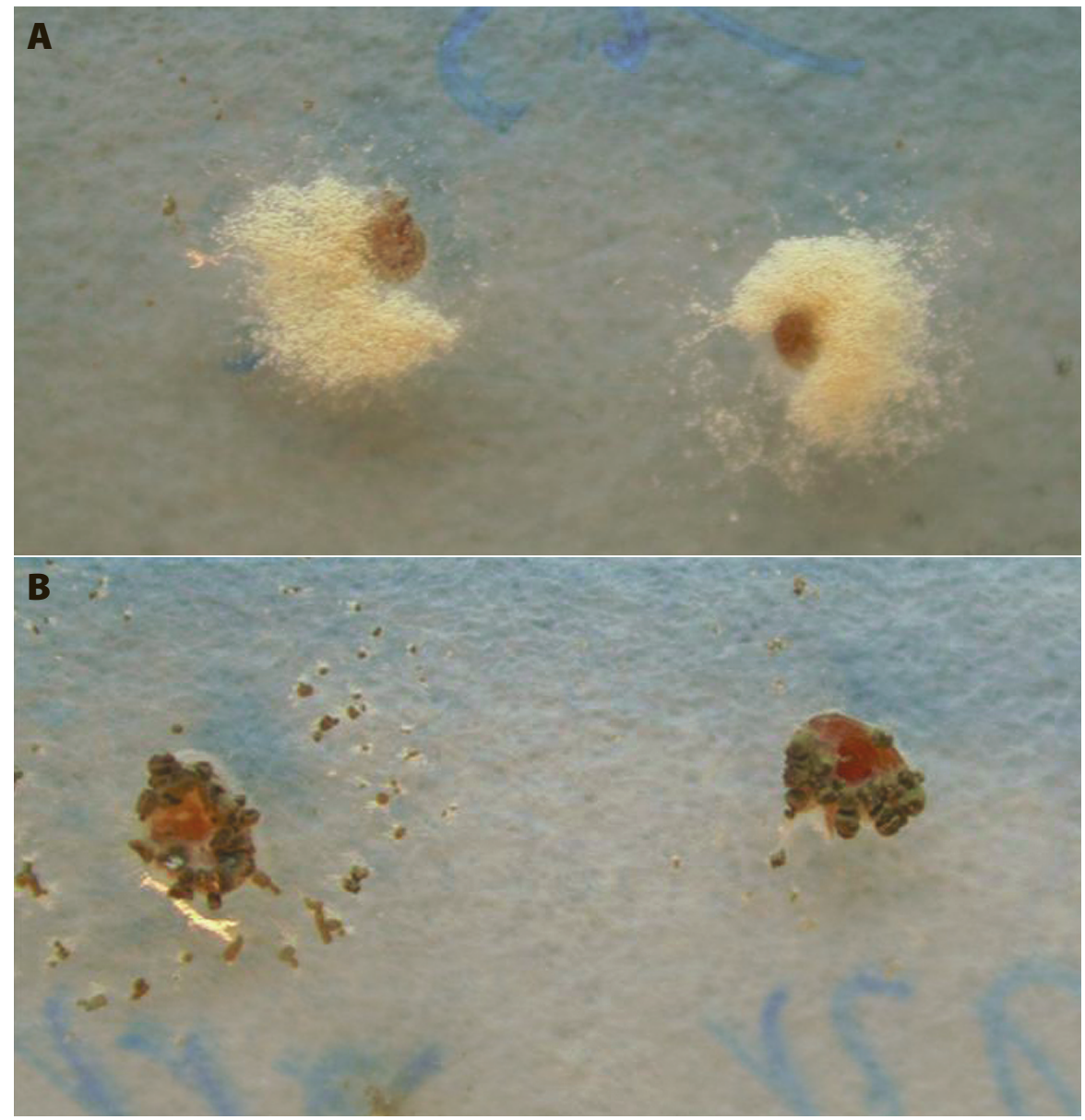

Isolates of $M$. anisopliae have been used to control many pests, including termites (Su et al., 2003) and locusts (Cherry et al., 1999). Beauveria bassiana is known to have a wide host range (Tanada and Kaya, 1993) and has been used against whiteflies (Islam et al., 2010) and mosquitoes (Farenhorst et al., 2009). Both species have been evaluated for use against acarines, including ixodid ticks (e.g., Stafford and Allan, 2011) and tetranychid mites (e.g., Bugeme et al., 2010). Beauveria bassiana is also, to date, the only EPF found naturally occurring on $V$. destructor, having been isolated from mites (see Figure 3) in Russia (Chernov, 1981 cited in Chandler et al., 2000), France (Meikle et al., 2006), Spain (GarcíaFernández et al., 2008), and Denmark (Steenberg et al., 2010).

Isolates of $M$. anisopliae have been evaluated against $V$. destructor by several research groups. Kanga et al. (2002, 2003, 2010) reported results of laboratory and field tests using $M$. anisopliae conidia introduced into the hives via several means, including strips, sprinkling between frames, and incorporated in protein patties. Kanga et al. (2003) reported good success in field trials, particularly when compared to tau-fluvalinate (Apistan ${ }^{\odot}$ ) but treated the hives with comparatively large amounts of material: $46.8 \mathrm{~g}$ of conidia powder (1010 conidia per gram) for the "low dose" treatment and twice that for the high dose. Given the cost of EPF conidia, it is questionable whether these dosage rates would be commercially feasible. Kanga et al. (2010) also reported good success using M. anisopliae conidia mixed with protein patties but did not include
Figure 4. A: Varroa infected with Beauveria fungus. B: Varroa infected with Metarhizium fungus. (Photos by G. Mercadier.) 
Figure 5. Larvae of the small hive beetle on the bottom board; when mature they will crawl outside to pupate in the soil. (Photo by D. Sammataro.)

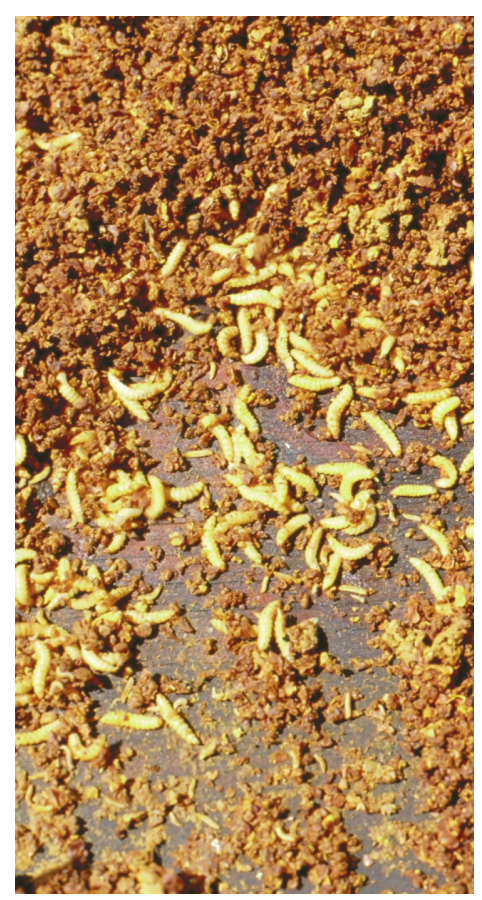

a "positive control" treatment to control for the effects of the protein patties themselves. Protein patties are a common hive treatment known to improve bee nutrition, increase worker bee production, and help colonies recover from diseases (Herbert, 1992). Thus, the results were inconclusive.

Other workers have reported equivocal results. James et al. (2006) tested M. anisopliae but without success. They tried a number of different application methods but observed no impact of treatment on mite densities and felt that the $M$. anisopliae conidia were possibly not coming into contact with enough $V$. destructor to reduce their density. Rodríguez et al. (2009a,b) first identified a desirable $M$. ansisopliae isolate based on temperature tolerances, then applied the conidia using several methods, including sprinkling the conidia between frames, attaching the conidia to strips hung between frames, and using an auto-application device at the entrance of the hive. While Rodríguez et al. (2009b) found that conidia sprinkled between frames gave the best results, bee mortality was highest in that treatment and, with respect to $V$. destructor, the biopesticide treatment needed further work to be considered a viable strategy.

Beauveria bassiana has not been tested as extensively as $M$. anisopliae. Meikle et al. (2007) mixed conidia of an isolate of B. bassiana collected from $V$. destructor in bee hives with carnauba wax powder and hydrated silica $\left(8-12 \times 10^{9}\right.$ conidia per $\left.g\right)$ and then applied that mixture between frames to control $V$. destructor. While high mortality rates of $V$. destructor and little or no impact on bee colony health were observed in treated hives, a single application did not sufficiently control the mite. Meikle et al. (2008a,b; 2009) reported further field experiments with different formulation ingredients (candelilla wax powder or wheat flour, or no ingredients at all), and in single or multiple applications. Some results were encouraging: Meikle et al. (2009) reported that, after three applications, mite fall onto sticky boards was much lower in treated hives than in untreated control hives. However, in that case, phoretic mite densities remained unacceptably high. A better understanding of the ecology of EPF in bee hives, particularly B. bassiana, was seen as crucial to designing more effective control strategies.

In summary, development of an effective biological control program against $V$. destructor will likely take the form of an effective biopesticide formulation and application method. In addition, questions such as why $B$. bassiana has been found associated with $V$. destructor and no other, how B. bassiana gains entrance to bee hives, and what happens to EPF conidia in the hive are in need of further research.

\section{Small Hive Beetle}

Some workers have explored the possibility of biological control of the Small Hive Beetle (SHB) (see Chapter 13 of this edition for a review of SHB biology and ecology). While many of the same agents used against $V$. destructor have also been tested against SHB in laboratory sessions, SHB has life history traits that make it a very different kind of pest. This might have implications on its control. First, unlike $V$. destructor, SHB is not an obligate bee parasite; larvae feed on and contaminate pollen and honey stores in addition to bee brood. Second, SHB must spend part of its life, specifically pupation, outside of the hive (see Figure 5) and adults can be found both inside (see Figure 6) outside the hive. Taken together, these characteristics offer both a challenge in that control of the beetles 


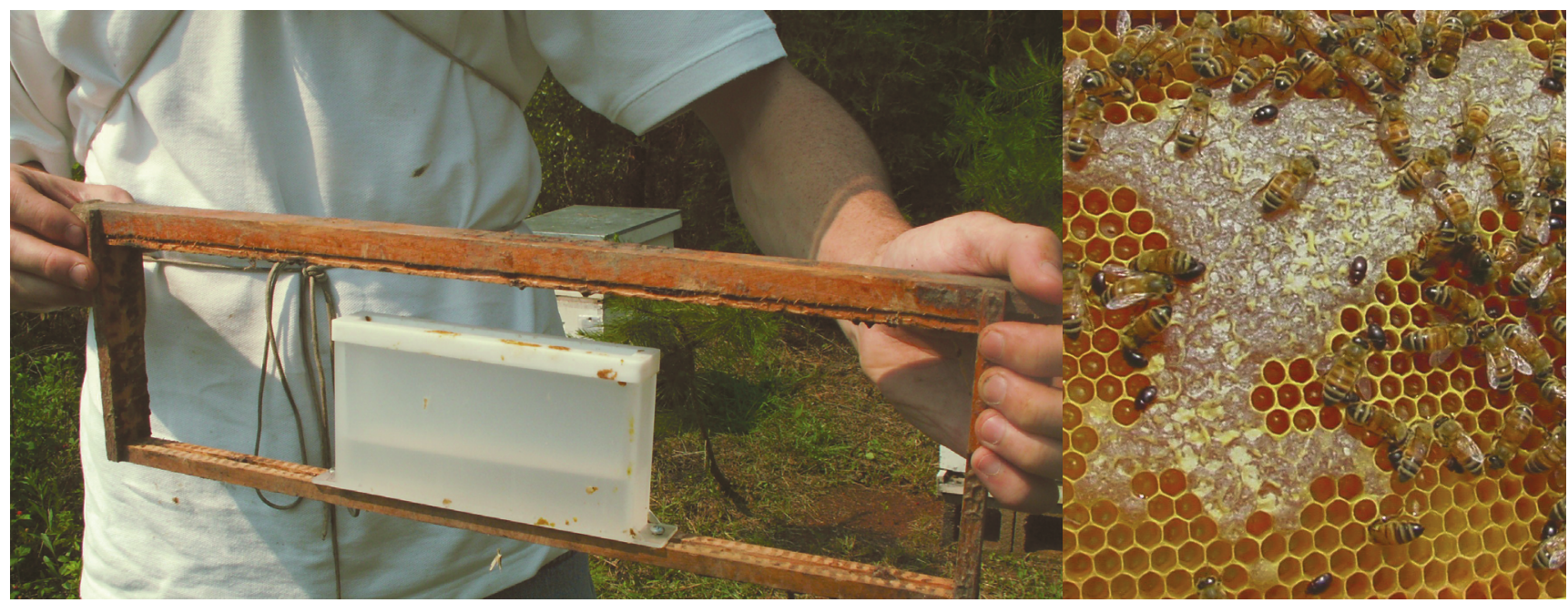

may involve controlling them outside as well as inside the hive, and an opportunity in that SHB control outside the hive may have fewer restrictions than in-hive control. Successful control outside the hive would be expected to impact the ability of SHB populations to attack hives in large numbers.

As with $V$. destructor, biological control options thus far have been limited to a predator, pseudoscorpions (Donovan and Paul, 2005), and pathogens, in the form of nematodes (Cabanillas and Elzen, 2006; Ellis et al., 2010; Shapiro-Ilan et al., 2010) and EPF (Ellis et al., 2004; Muerlle et al., 2006; Richards et al., 2005). In the case of SHB, which spends different life stages in different environments, the choice of a biological control agent also rests on the target stage of the insect. Pseudoscorpions, which are expected to live inside the hive, would be either unlikely or unable to attack wandering larvae or pupae because they are buried in the soil. Likewise, adult beetles might not be suitable prey because they are active and have tough elytra. However, pseudoscorpions could attack eggs and young larvae in the hive. That would be highly desirable because the larvae would not have had a chance to cause much damage. To our knowledge, no field experiments have been conducted with biocontrol agents against SHB.

The use of nematodes would target SHB either in the wandering larvae stage or in pupation in the soil. Cabanillas and Elzen (2006), Ellis et al. (2010), and Shapiro-Ilan et al. (2010) all conducted experiments exploring the use of nematodes against SHB in the soil environment. Targeting the wandering larval and pupal stages of SHB removes difficulties such as bees being attacked by the nematodes, or bees removing the nematodes while cleaning. However, that method targets the beetles after they have done damage as larvae; its effectiveness will depend on factors such as the degree to which emerging beetles attack the hive from which they originated. A single small hive beetle can lay thousands of eggs (Ellis et al., 2002; Meikle and Patt, 2011), so even a few females would have the potential to cause serious problems for a weak or small hive that cannot maintain proper hygiene.

Finally, EPF have been explored as biological control agents of SHB. Ellis et al. (2004) and Richards et al. (2005) examined Aspergillus niger (van Tieghem) and A. flavus (Link: Grey) (both Eurotiaceae) as potential soil treatments against pupal SHB. Both teams of researchers found
Figure 6. A Hood beetle trap positioned in a medium frame. The trap can be provisioned with a bait (such as apple cider vinegar) and a killing agent (mineral oil). Adult beetles (like those on the frame in the picture on the right) will enter the trap and be exposed to the killing agent. (Photos by University of Florida. Used with permission.) 
significant treatment effects where healthy larvae or pupae were exposed to the pathogens. However, as Ellis et al. (2004) noted, fungi of the genus Aspergillus are known to produce mycotoxins. While the potential for mycotoxin contamination from a human's point of view must be addressed, from the point of view of a honey bee the situation may be different. Niu et al. (2010) reported that propolis (bee-collected plant resins) helped bees break down mycotoxins such as those found in bee bread by enhancing the activity of enzymes involved in detoxification. An additional and possibly bigger problem with respect to Aspergillus fungi is that they can cause bee disease, including stonebrood (Muerle et al. 2006).

Muerrle et al. (2006) examined several commonly used EPF, including M. anisopliae, B. bassiana, and Hirsutella illustris (another hypocrealean EPF) for their effect on SHB in laboratory bioassays. Muerrle et al. (2006) collected an isolate of $M$. anisopliae from SHB in South Africa but found that the most virulent isolate in their tests was a B. bassiana isolate collected from termites.

\section{Conclusion}

Biological control of bee pests is still largely unexplored but interest is growing as beekeepers and bee researchers try to develop integrated methods of bee pest management and reduce the use of chemicals. Effective biological control methods have been developed for wax moth, but their use is apparently limited, probably owing to the lesser importance of wax moth compared to other honey bee threats. Results of Varroa biological control have been variable and limited to EPF; at the very least, further work is needed on identifying appropriate isolates and on developing appropriate formulations and application methods. In addition, field testing of SHB biological controls is needed to improve our understanding of SHB ecology. 